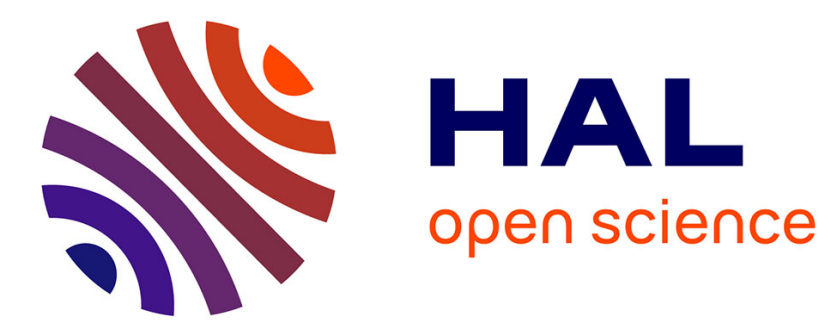

\title{
Observation simultanée de deux groupes de raies d'un spectre de masse
}

\author{
M. Becourt
}

\section{To cite this version:}

M. Becourt. Observation simultanée de deux groupes de raies d'un spectre de masse. Revue de Physique Appliquée, 1968, 3 (3), pp.222-224. 10.1051/rphysap:0196800303022201 . jpa-00242851

\section{HAL Id: jpa-00242851 https://hal.science/jpa-00242851}

Submitted on 1 Jan 1968

HAL is a multi-disciplinary open access archive for the deposit and dissemination of scientific research documents, whether they are published or not. The documents may come from teaching and research institutions in France or abroad, or from public or private research centers.
L'archive ouverte pluridisciplinaire HAL, est destinée au dépôt et à la diffusion de documents scientifiques de niveau recherche, publiés ou non, émanant des établissements d'enseignement et de recherche français ou étrangers, des laboratoires publics ou privés. 


\title{
BIBLIOGRAPHIE
}

[1] LEE et PAPAS, Radiation resistance and irreversible power of antennas in gyroelectric media, IEEE Trans. Ant. Prop., A.P. 13, 5, 834-835 ; Irreversible power and radiation resistance of antennae in anisotropic ionized gases, Radio Science J. Res., NBS 69D, 10, 1313-1320 ; A further explanation of the new theory of antenna radiation with particular reference to uniaxial media, Radio Science J. Res., sept. 66, 1, 9, 1020-1023.

[2] STARAS, The "infinity catastrophe" associated with radiation in magnetoionic media, Radio Science $J$. Res., sept. 66, 1, 9, 1013-1020.
[3] WALSH (D.) et WEIL (H.), Irreversible power and radiation resistance of antenna in magnetoionic media, Radio Science J. Res., sept. 66, 1, 9, 10251027.

[4] KOGEINIK (H.), On electromagnetic radiation in magnetoionic media, J. Res. Nat. Bur. Stand., sept.-oct. 1960, 64 D, 5 .

[5] LAfFON (J. P.) et WEIL (H.), Impedance of "cylindrical" antennas in magnetoplasma under conditions of refractive index resonance. A paraître.

[6] LAFON (J. P.), Condition de rayonnement-Condition d'antirayonnement, 1968.

\section{OBSERVATION SIMULTANÉE DE DEUX GROUPES DE RAIES D'UN SPEGTRE DE MASSE}

\author{
Par M. BEGOURT, \\ Laboratoire de Physique des Milieux Ionisés, École Polytechnique, Équipe de Recherche associée au G.N.R.S.
}

(Reçu le 9 avril 1968.)

Résumé. - Nous décrivons ici un générateur de tension avec ses circuits associés qui permet de balayer deux raies ou groupes de raies quelconques sur un spectromètre de masse, et de visualiser l'évolution de leurs amplitudes en fonction du temps. Le signal de sortie de la base de temps est la somme d'une tension continue d'une tension en dent de scie à la fréquence $f$, et avec la même phase, d'une tension carrée à la fréquence $f / 2$.

Abstract. - A special power supply and its associated circuits are described. The output voltage is applied to a mass spectrometer in order to sweep two lines or any group of lines and to depict the variations of the amplitudes versus time on an oscilloscope. The waveshape of the time base is the sum of a direct current voltage, a sawtooth of frequency $f$ and with the same phase, and a square wave of frequency $t / 2$.

I. Introduction. - Un analyseur de spectre permet d'obtenir un déroulement continu du spectre de masse, entre des limites fixées au préalable. Ce déroulement est d'autant plus lent que le spectre à observer est plus large, et s'il permet parfaitement l'enregistrement graphique, il est très difficile de comparer sur un oscilloscope deux raies ou groupes de raies, de masses et d'intensités très différentes.

Pour remédier à cet inconvénient, nous avons réalisé une base de temps qui, associée à un analyseur « Veeco GA-4 » et à un oscilloscope « Tektronix 561-A», permet d'observer deux parties quelconques du spectre, de largeur variable, sur chacune des traces d'un tiroir 3A3, et de comparer simultanément les amplitudes respectives des masses, même si celles-ci sont très différentes en choisissant la sensibilité de chacune des voies.

II. Fonctionnement de l'analyseur « Veeco GA-4 ». Le tube analyseur, qui est l'élément sensible du spectromètre de masse, requiert une alimentation haute tension variable, un amplificateur électrométrique à liaison directe et un organe de lecture du type oscilloscope ou enregistreur.

Pour balayer tout le spectre, il faut, quel que soit le mode de fonctionnement utilisé : manuel ou automatique, appliquer à l'amplificateur qui commande le tube un signal variable de 0 à $150 \mathrm{~V}$. Par suite de 
ses caractéristiques techniques, l'ensemble de l'analyseur ne permet que des variations lentes, d'où l'impossibilité de comparer simultanément les variations d'amplitude de deux ou plusieurs raies de masses et d'amplitudes différentes.

Pour pratiquer ces mesures, la méthode suivante est proposée.

Principe. - Pour réaliser cette manipulation, nous avons associé un spectromètre de masse « Veeco type GA-4 » avec un oscilloscope « Tektronix 561-A » équipé pour sa partie verticale d'un tiroir $3 \mathrm{~A} 3$ et sur sa partie horizontale d'un ensemble électronique conçu spécialement, qui permet :

- de délivrer le signal d'excitation nécessaire au fonctionnement du spectromètre de masse,

- de fournir les signaux de déclenchement de la trace alternée, d'extinction et de balayage horizontal du tube cathodique.

Le signal d'excitation, dont la forme est déterminée par la superposition d'un signal en dent de scie et d'un signal rectangulaire synchronisé, est appliqué à l'analyseur de spectre mis préalablement sur la position manuelle.

Tous les paramètres sont réglables et la fréquence pourra être d'autant plus élevée que la déviation en balayage sera plus faible.

Ainsi, ce signal permet de passer rapidement de l'analyse d'une raie ou d'un groupe à une autre raie ou à un autre groupe, sans balayer toutes les masses intermédiaires.

A la sortie de l'amplificateur de lecture sortent alter-

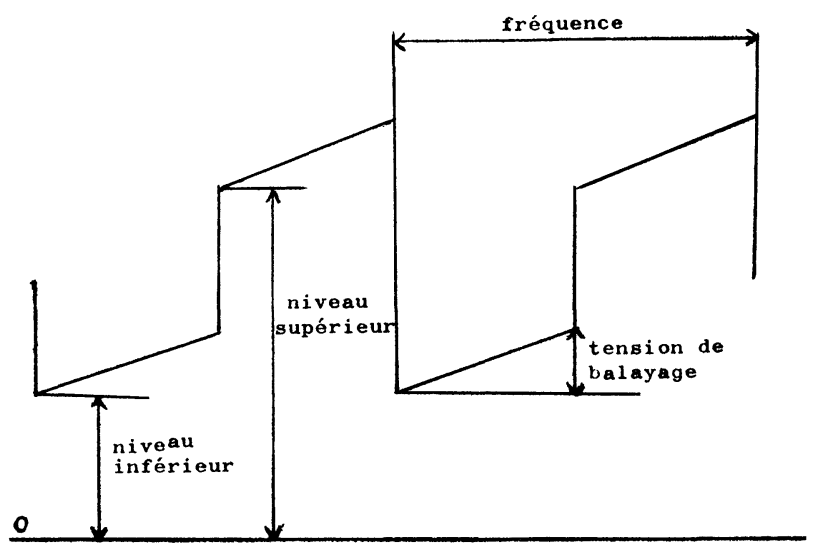

FORME DU SIGNAL.

nativement les signaux dus au balayage inférieur et ceux dus au balayage supérieur.

Ces signaux sont appliqués sur l'entrée verticale de l'oscilloscope 561-A qui est équipé d'un tiroir possédant un balayage alterné synchronisé avec les signaux de déflexion horizontale du tube cathodique (fig. 1).

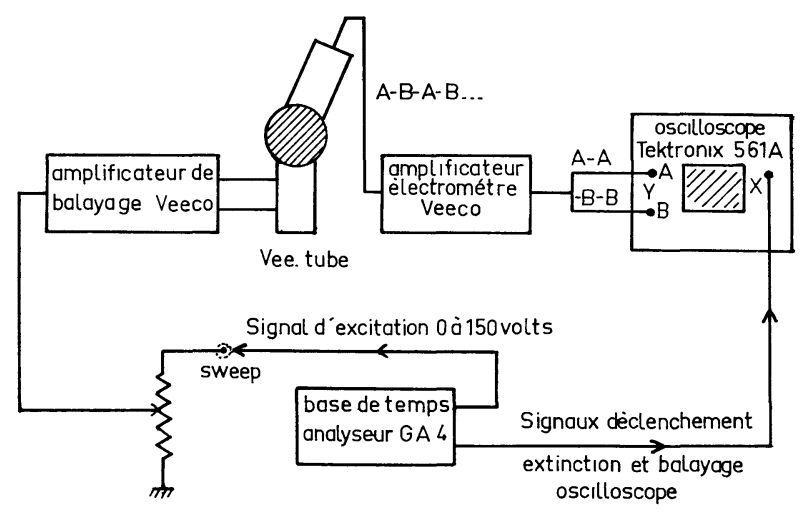

FIG. 1. - Spectrométrie de masse :

schéma de principe avec adjonction de la base de temps.

III. Circuits électroniques. — La base de temps pour analyseur GA-4 comprend :

- la fabrication du signal d'excitation;

- les circuits d'extinction, de déclenchement et de balayage horizontal du tube cathodique.

A. Fabrication du signal D'excitation. - Le signal d'excitation est l'addition d'un signal en dent de scie variable en amplitude de 0 à $70 \mathrm{~V}$ et en période de 1,2 à $20 \mathrm{~s}$, et d'un signal rectangulaire synchronisé par la dérivation du front arrière du signal en dent de scie, variable en amplitude et en niveau continu par rapport à la masse de 0 à $+150 \mathrm{~V}$.

La tension en dent de scie est également reliée au point commun d'un second générateur qui délivre les créneaux rectangulaires et fonctionne de la façon suivante :

Une résistance $R_{1}$ alimentée à travers une diode $D_{2}$ recueille à ses bornes une tension égale en amplitude (à la chute de tension près de la diode) à celle affichée

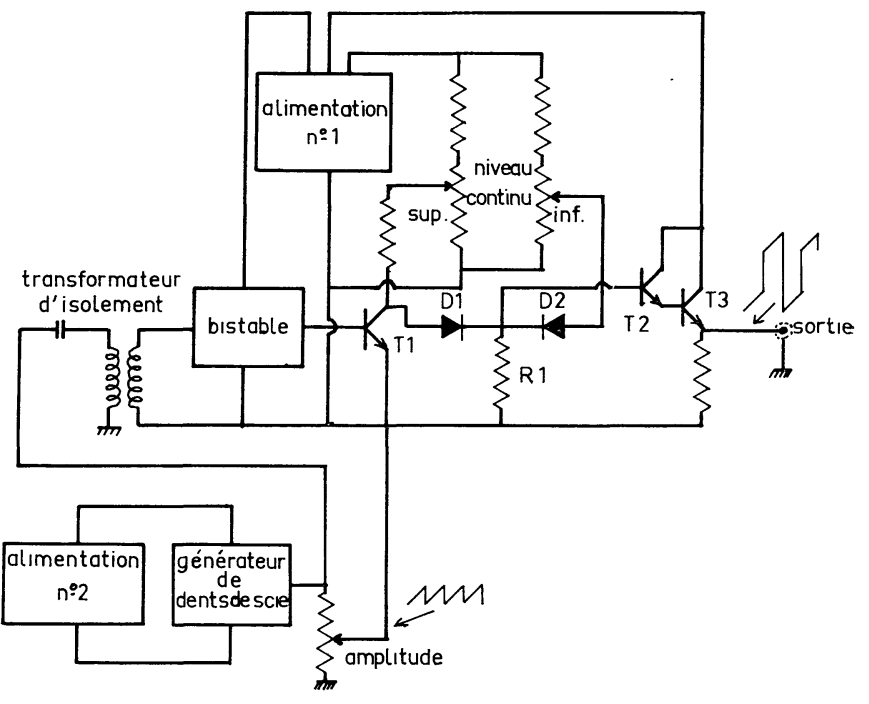

FIG. 2.

Synoptique de fabrication du signal d'excitation. 
par le curseur du potentiomètre 10 tours " niveau continu inférieur » lorsque le transistor $T_{1}$ (MJ 421) commandé par le bistable est conducteur, et une tension égale à celle affichée par le curseur du potentiomètre " niveau continu supérieur » lorsque $T_{1}$ est bloqué. Par construction, $V_{1}$ ne peut jamais être inférieur à $V_{2}$. Le transistor $T_{1}$ est alternativement conducteur ou bloqué en synchronisme avec le générateur en dent de scie qui, par l'intermédiaire d'un transformateur d'isolement, attaque une bascule bistable, laquelle polarise $T_{1}$ en régime saturé ou bloqué.

Le signal aux bornes de $R_{1}$ est repris par un montage Darlington et permet de sortir à basse impédance (voir le schéma synoptique de fabrication du signal d'excitation, fig. 2).

B. Cirguit de balayage horizontal et déclenGHEMENT DE LA TRAGE ALTERnÉE. - 1 . Circuit de balayage horizontal. - L'amplificateur de balayage horizontal est un amplificateur différentiel couplé au générateur d'excitation, de façon à être en synchronisme avec lui par l'intermédiaire d'un étage permettant de régler le niveau continu sur lequel est superposé le signal.

2. Déclenchement de la trace alternée. - Un trigger de Schmitt synchronisé sur la montée du signal en dent de scie permet :

a) De déclencher la bascule de la trace alternée sur le tiroir vertical;

b) De délivrer une impulsion pour éteindre le retour de balayage.

Ces deux signaux sont délivrés par une bascule monostable.

IV. Exemple d'utilisation. - Un balayage normal sur la position automatique est effectué avec une vitesse de déroulement égale à $10 \mathrm{mn}$ pour tout le spectre, sur l'échelle de sortie 300 .

Un autre balayage par le système proposé a été photographié et est délimité sur la courbe d'enregistrement normal :

1) Dans la méthode de fonctionnement classique (fig. 3), on voit que l'importance de la raie 18 ne permet pas d'observer, si l'on ne sature pas l'amplificateur de sortie, les raies $26,27,29$ et 32 du fait de leur faible amplitude.
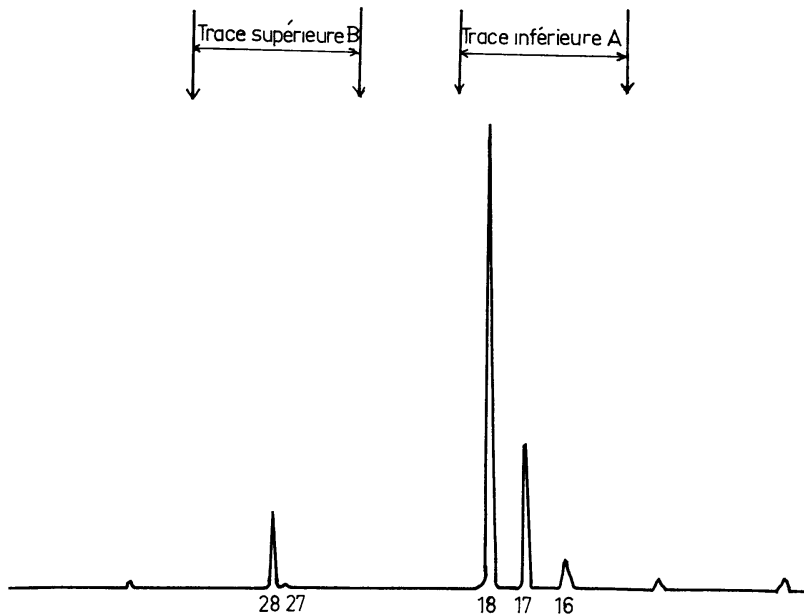

Fig. 3. - Balayage du spectre par la méthode classique. Relevé de l'enregistreur.

Le réglage des sensibilités de l'oscilloscope sur chaque entrée permet l'observation simultanée de ces raies sans saturation.

2) Seules les zones de balayage intéressantes sont visualisées, avec une période minimum récurrente de $20 \mathrm{~s}$, ce qui permet la comparaison simultanée des raies du spectre contenu dans les deux groupes ( fig. 4).

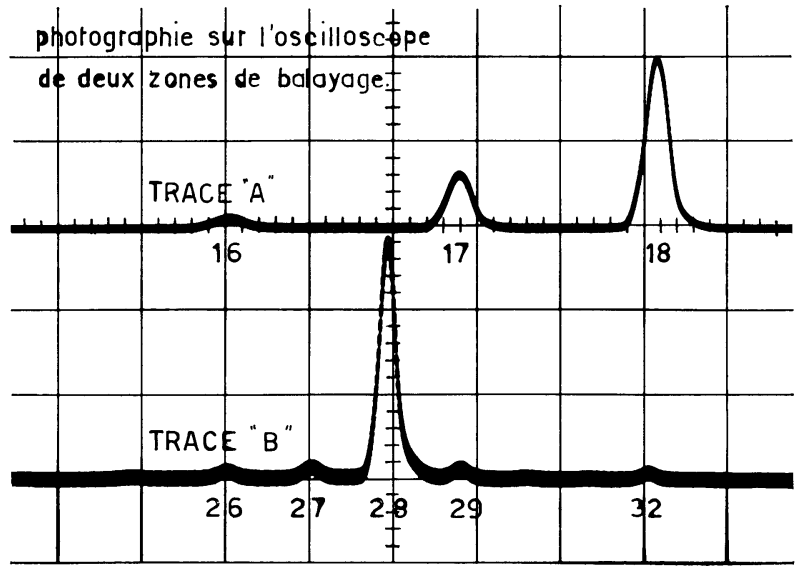

FIG. 4. - Balayage en deux zones avec la base de temps Photographie prise sur l'oscilloscope. 